\title{
Investigation into the damping and stiffness characteristics of an elevator car system
}

\author{
I. Herrera ${ }^{1, a}, \mathrm{H} . \mathrm{Su}^{2, b}$ and S. Kaczmarczyk ${ }^{2, \mathrm{c}}$ \\ ${ }^{1}$ Escuela de Ingenierías Industriales, Universidad de Extremadura, Avda. de Elvas s/n, 06071 \\ Badajoz, Spain \\ ${ }^{2}$ School of Applied Sciences, University of Northampton, St. George's Avenue, Northampton, NN2 \\ 6JD, UK \\ aiherrera@unex.es, bhuijuan.su@northampton.ac.uk, 'stefan.kaczmarczyk@northampton.ac.uk
}

Keywords: Stiffness coefficient, damping coefficient, elevator car system

\begin{abstract}
Modelling the dynamic performance of an elevator car system represents a complex task and forms an important step in the elevator system design procedure. The need to consider the behaviour of passengers travelling in the car complicates the procedure further. This paper presents an original approach to identify the stiffness and damping characteristics of an elevator car system. A simplified model is developed and the experimental rig with a rectangular elevator platform fixed on the top of four silent blocks attached to a shaker is setup. The transmissibility measurements are carried out with a harmonic excitation applied first to a platform with no passenger load and then to the platform with one passenger within the frequency range of $1-20 \mathrm{~Hz}$. A single person standing on the platform is employed in order to assess the passenger's contribution to the dynamic behaviour of the elevator car system. The curve fitting technique implemented in MATLAB is used to determine the damping and stiffness coefficients both for the empty car system and the carpassenger system. Investigation on the tolerances for both parameters is carried out. An approach to simplify the experimental procedure and to reduce the number of individual tests is proposed.
\end{abstract}

\section{Introduction}

The dynamic characteristics of the elevator car system play a very important role in elevator engineering. Nowadays, high ride quality of elevators is demanded. In particular low vibration and noise levels are related to high ride quality. These are usually checked by elevator companies before launching a new product into market by experiments and/or modelling. Modelling the dynamic performance of an elevator car system is a complex task and forms a significant step in the elevator system design procedure. As stated in the ISO standard [1] for elevator ride quality measurements, the components affecting the acceleration measurements in the car system are the instrument, persons and floor covering. Much effort has been made to predict passenger's behaviour and characteristics on the dynamic response of an elevator car [2,3,4]. In the previous investigations [2] passengers were modelled as rigid bodies with different types of shoes and it was concluded that the dynamic response of the elevator car could be substantially influenced by the passenger load. The need to consider the dynamics of passengers riding in the car complicates the procedure further $[5,6]$.

In this paper the results of a study to assess the influence of the dynamic characteristics of a passenger on the lift car response are presented. The transmissibility measurements have been carried out. The curve fitting toolbox implemented in MATLAB [7] is then used in order to identify the stiffness and damping characteristics of the elevator car-passenger system. An approach is proposed to simplify the experimental procedure by reducing the number of transmissibility measurements by focusing on the resonance frequency region rather than using the whole range of the test frequencies. 


\section{Mechanical Model of the Car System}

A typical elevator car system consists of the cabin (car enclosure) and the car frame (see Fig. 1). The cabin carries passengers and the car frame is connected to the means of suspension and to the platform (the floor) which directly supports the passengers. Considering vertical only, a simplified two degree of freedom model as shown in Fig. 2 is used to represent the car-passenger system. The model includes a single passenger represented by a rigid mass $\mathrm{m}_{\mathrm{PA}}$. The passenger is coupled to the platform via a spring-damper element of coefficient of stiffness $\mathrm{k}_{\mathrm{PA}}$ and of coefficient of viscous damping $\mathrm{c}_{\mathrm{PA}}$, respectively. The car enclosure is modelled as a rigid mass $\mathrm{m}_{\mathrm{CA}}$. The platform is isolated from the frame by four isolation blocks represented by a spring-damper element of stiffness coefficient $\mathrm{k}_{\mathrm{CA}}$ and of viscous damping coefficient $\mathrm{c}_{\mathrm{CA}}$, respectively. The car frame is subjected to a harmonic base motion excitation $\mathrm{y}_{\mathrm{FR}}$. The vertical displacements of the passenger and the car enclosure are denoted as $\mathrm{y}_{\mathrm{PA}}$ and $\mathrm{y}_{\mathrm{CA}}$, respectively.

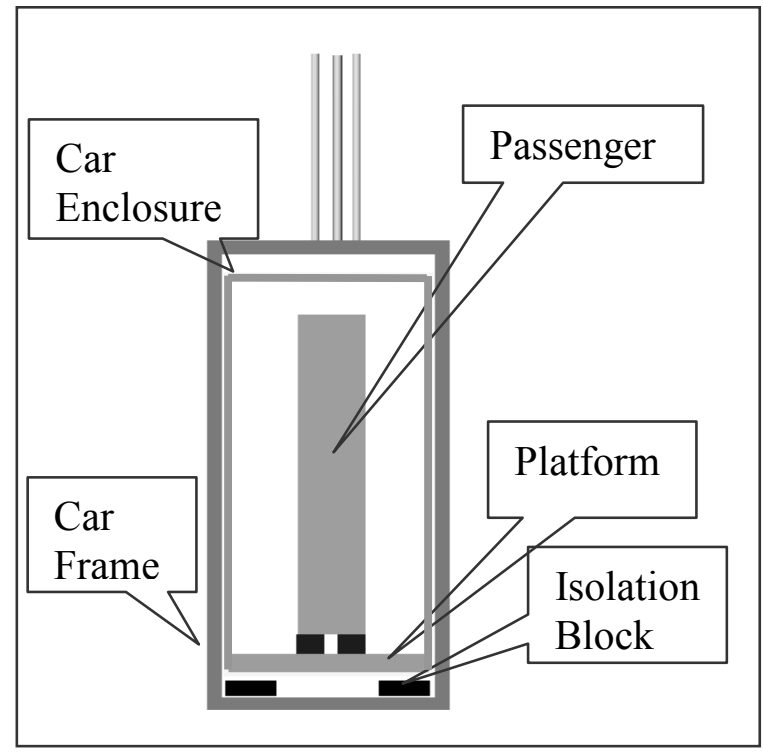

Figure 1. The car-passenger subsystem

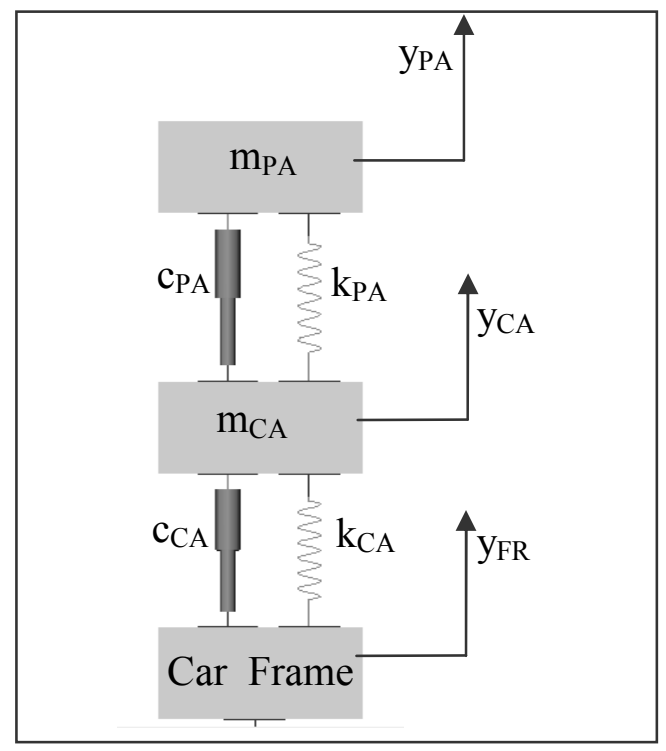

Figure 2. A model of the car-passenger system

The coefficients $\mathrm{k}_{\mathrm{CA}}$ and $\mathrm{c}_{\mathrm{CA}}$ are determined as [2],

$$
k_{C A}=n k_{s p} ; \quad c_{C A}=n c_{s p}
$$

where $\mathrm{k}_{\mathrm{sp}}$ and $\mathrm{c}_{\mathrm{sp}}$ are the coefficients of stiffness and damping of a single isolation block, respectively, and $n$ is the number of isolation blocks used.

The coefficients $\mathrm{k}_{\mathrm{PA}}$ and $\mathrm{c}_{\mathrm{PA}}$ are then defined as

$$
\frac{1}{k_{P A}}=\frac{1}{k_{p a}}+\frac{1}{k_{s h}}+\frac{1}{k_{f l}} ; \quad \frac{1}{c_{P A}}=\frac{1}{c_{p a}}+\frac{1}{c_{s h}}+\frac{1}{c_{f l}}
$$

where $\mathrm{k}_{\mathrm{pa}}$ and $\mathrm{c}_{\mathrm{ca}}$ denote the stiffness and damping coefficients of a human body in the vertical direction, respectively, $\mathrm{k}_{\mathrm{sh}}$ and $\mathrm{c}_{\mathrm{sh}}$ are the vertical stiffness and damping coefficients of the passenger's shoes, and $\mathrm{k}_{\mathrm{fl}}$ and $\mathrm{c}_{\mathrm{fl}}$ represent the vertical stiffness and damping coefficients of the platform/floor covering. 
The differential equation of motion of the car-passenger system in matrix form is given as:

$$
\left[\begin{array}{cc}
m_{C A} & 0 \\
0 & m_{P A}
\end{array}\right]\left[\begin{array}{c}
\ddot{y}_{C A} \\
\ddot{y}_{P A}
\end{array}\right]+\left[\begin{array}{cc}
c_{C A}+c_{P A} & -c_{P A} \\
-c_{P A} & c_{P A}
\end{array}\right]\left[\begin{array}{c}
\dot{y}_{C A} \\
\dot{y}_{P A}
\end{array}\right]+\left[\begin{array}{cc}
k_{C A}+k_{P A} & -k_{P A} \\
-k_{P A} & k_{P A}
\end{array}\right]\left[\begin{array}{c}
y_{C A} \\
y_{P A}
\end{array}\right]=\left[\begin{array}{c}
c_{C A} \dot{y}_{F R}+k_{C A} y_{F R} \\
0
\end{array}\right]
$$

In order to identify the resonance frequency, a frequency sweep is applied to investigate the forced response of the system. The sinusoidal frequency sweep is applied as

$$
y_{F R}(\tau)=y_{0} \sin (\Omega \tau)
$$

where $y_{0}$ is the amplitude and

$$
\Omega=\omega / \omega_{n C A}
$$

where $\omega$ is the angular frequency of the shaker excitation and $\tau$ represents the non-dimensional time defined as $\tau=\omega_{n C A} t$.

The non-dimensional parameters are defined as

$$
\omega_{n C A}^{2}=\frac{k_{C A}}{m_{C A}} ; \omega_{n P A}^{2}=\frac{k_{P A}}{m_{P A}} ; m_{r}=\frac{m_{P A}}{m_{C A}} ; \omega_{r}=\frac{\omega_{n P A}}{\omega_{n C A}} ; 2 \xi_{C A}=\frac{c_{C A}}{m_{C A} \omega_{n C A}} ; 2 \xi_{P A}=\frac{c_{P A}}{m_{P A} \omega_{n P A}}
$$

Introducing the non-dimensional parameters and time $\tau$ into Eq. (3), computing the Laplace transform and transposing yields the transfer function (the displacement transmissibility)

$$
\begin{aligned}
& G(\Omega)=\left|\frac{Y_{C A}(\Omega j)}{Y_{F R}(\Omega j)}\right| \\
& =\left|\frac{\left(1+2 j \xi_{C A} \Omega\right)\left(\omega_{r}^{2}-\Omega^{2}+2 j \xi_{P A} \omega_{r} \Omega\right)}{\left(\Omega^{4}-\left[\left(\omega_{r}^{2}+2 j \xi_{P A} \omega_{r} \Omega\right)\left(1+m_{r}\right)+\left(1+2 j \xi_{C A} \Omega\right)\right] \Omega^{2}+\left(1+2 j \xi_{C A} \Omega\right)\left(\omega_{r}^{2}+2 j \xi_{P A} \omega_{r} \Omega\right)\right.}\right|
\end{aligned}
$$

In the case of an empty car system, Eq. (7) is reduced to a single degree of freedom model and the displacement transmissibility assumes the following form

$$
G^{1}(\Omega)=\left|\frac{1+2 j \xi_{C A} \Omega}{1-\Omega^{2}+2 j \xi_{C A} \Omega}\right|
$$

\section{Experimental Setup}

The experimental rig has been developed to carry out the testing programme. The rig employed a rectangular $110 \mathrm{~cm}$ by $106 \mathrm{~cm}$ platform of mass $202 \mathrm{~kg}$ fixed on the top of four silent blocks. The platform was then excited by a shaker actuator. The CSI 2130 portable analyzer was used to carry out data acquisition and signal analysis. Two IMI-ICP 626A04 piezoelectric accelerometers, designed for low frequency usage, were fixed to the actuator table and to the rigid base of the car platform. First, the acceleration transmissibility was measured using a harmonic excitation applied to the platform with no passenger load within the frequency range from 1 to $20 \mathrm{~Hz}$. This facilitated the identification of the resonance frequency (denoted as $f_{r}$, see Fig. 3) and the damping ratio $\xi_{\mathrm{CA}}$ using Eqs. (5) and (8) in order to determine the coefficients of stiffness $\mathrm{k}_{\mathrm{CA}}$ and damping $\mathrm{c}_{\mathrm{CA}}$, respectively. Next, the acceleration transmissibility corresponding to the platform carrying a 
passenger of mass $\mathrm{m}_{\mathrm{PA}}=80 \mathrm{~kg}$ was measured and the experimental data were used to determine the effective coefficient of stiffness $\mathrm{k}_{\mathrm{PA}}$ and the effective damping coefficient $\mathrm{c}_{\mathrm{PA}}$ of the passenger by using the MATLAB curve fitting toolbox [7].

\section{Results and Discussions}

The curve fitting technique employing the nonlinear least square method together with the Trustregion algorithm is applied to determine the damping and stiffness coefficients both corresponding to the empty car system (no passenger load) and to the car-passenger system with one passenger load. First the method is used to determine the resonance frequency $\left(f_{r}\right)$ and the damping ratio $\left(\xi_{\mathrm{CA}}\right)$ of the empty car system. The experimental transmissibility measurements for the car system with no passenger load are shown in cross points in Fig. 3. The data is fitted using the displacement transmissibility Eq. (8) together with Eq. (5) for the two unknowns, $f_{\mathrm{r}}$ and $\xi_{\mathrm{CA}}$ respectively. The fitted curve is shown in Fig. 3 as a solid line together with two thinner lines representing the fitted curves with $95 \%$ observation prediction bounds, which define the lower and upper values of the width of the interval. In other words, this indicates that there is a $95 \%$ chance that the observation is actually contained within the lower and upper prediction bounds. In order to determine whether the best fit is achieved, the numerical fits are required to be examined. The curve fitting toolbox in MATLAB supports the goodness-of-fit statistics for parametric models. They are the sum of squares due to error (SSE), R-square and root mean squared error (RMSE). These values for the empty car system are obtained as $0.666,0.9954$ and 0.2961 , respectively. The value of 0.9954 of R-square means that the fit explains $99.54 \%$ of the total variation in the data about the average. Considering all three values of statistics, it indicates a good fit achieved.

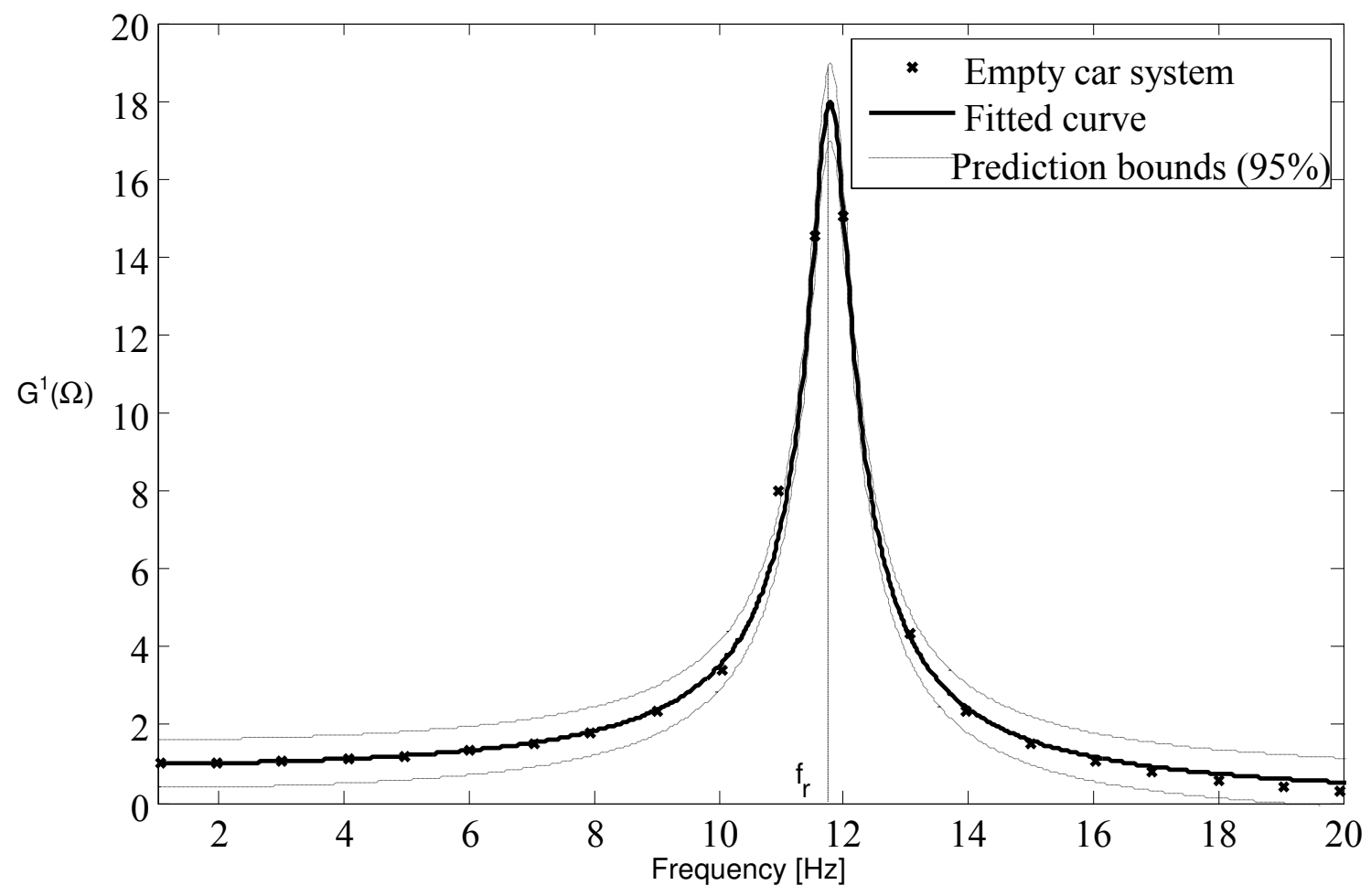

Figure 3. Fitted curves for the empty car system

As the confidence intervals on the results determine the accuracy, the two coefficients are obtained with $95 \%$ confidence bounds. The resonance frequency $\left(f_{r}\right)$ is identified as $11.79 \mathrm{~Hz}$ having the lower and upper bounds $11.77 \mathrm{~Hz}$ and $11.81 \mathrm{~Hz}$ with the interval width 0.04 . The damping ratio 
$\left(\xi_{\mathrm{CA}}\right)$ is found to be 0.02782 having the lower upper bounds 0.02661 and 0.02903 , respectively, with the interval width 0.00242 . Having obtained the parameters $\mathrm{f}_{\mathrm{r}}$ and $\xi_{\mathrm{CA}}$, the coefficients of stiffness $\left(\mathrm{k}_{\mathrm{CA}}\right)$ and damping $\left(\mathrm{c}_{\mathrm{CA}}\right)$ of the car system are calculated from Eq. (6) as $1108.5 \mathrm{kN} / \mathrm{m}$ and $832.59 \mathrm{~kg} / \mathrm{s}$ respectively.

The tests are then carried out with the single passenger load on the platform within the frequency range of $1-20 \mathrm{~Hz}$. The acceleration transmissibility is measured and the curve fitting technique is applied to the experimental data. Note the same procedure for the curve fitting is employed but Eq. (7) representing the two degree of freedom model taking into account the contribution of the passenger is now applied. The measured values of the transmissibility against the frequency range are shown in cross points in Fig. 4. Having identified the coefficients $\xi_{\mathrm{CA}}$ and $\mathrm{k}_{\mathrm{CA}}$ of the platform from the single degree of freedom model, the damping ratio $\xi_{\mathrm{PA}}$ and the stiffness coefficient $\mathrm{k}_{\mathrm{PA}}$ representing damping and stiffness characteristics of the passenger are determined using the curve fitting toolbox. The coefficients obtained using the data from the full frequency range are shown in Table 1 . With the $95 \%$ confidence bounds, the stiffness coefficient $\mathrm{k}_{\mathrm{PA}}$ is identified as $115.6 \mathrm{kN} / \mathrm{m}$ with the lower and upper bounds of $105.6 \mathrm{kN} / \mathrm{m}$ and $125.5 \mathrm{kN} / \mathrm{m}$, respectively. The error difference is calculated as $\pm 11.24 \%$. The damping ratio is 0.421 with the lower and upper bounds 0.3736 and 0.4684 , respectively. The error difference is $\pm 11.26 \%$. The damping coefficient $\left(\mathrm{c}_{\mathrm{PA}}\right)$ of the passenger is calculated using Eq. (6) as $2560.6 \mathrm{~kg} / \mathrm{s}$.

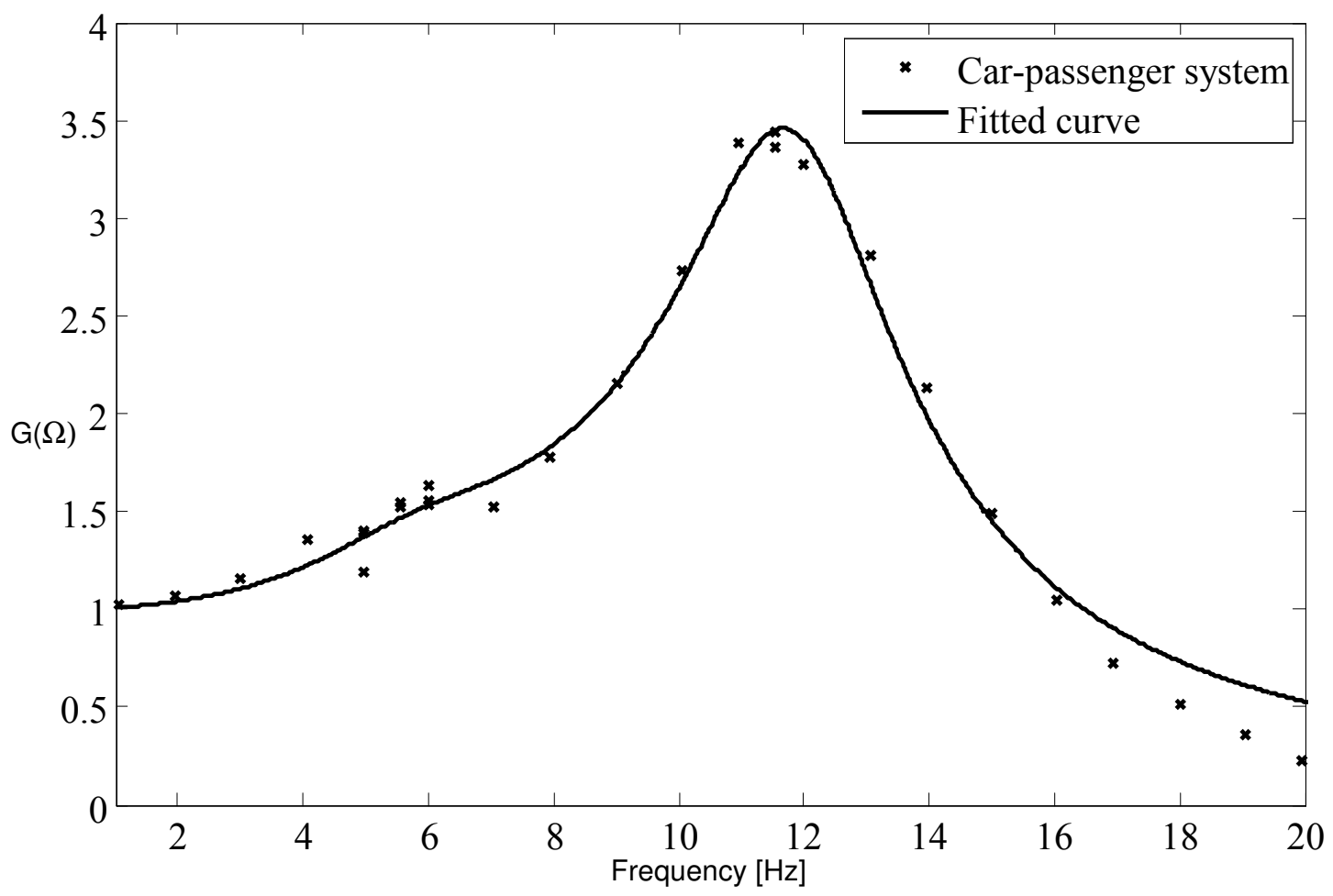

Figure 4. Fitted curve for the car-passenger system

Using reduced experimental data concentrated on the frequency range from 8-14 Hz, i.e. around the second resonance frequency, the two coefficients of the passenger are determined using the curve fitting technique. The results together with the statistics are shown in the third column in Table 1. With the $95 \%$ confidence bounds, the stiffness coefficient $\mathrm{k}_{\mathrm{PA}}$ is $115.9 \mathrm{kN} / \mathrm{m}$ with the lower and upper bounds $106.3 \mathrm{kN} / \mathrm{m}$ and $125.5 \mathrm{kN} / \mathrm{m}$. The error difference is $\pm 8.28 \%$. The damping ratio $\xi_{\mathrm{CA}}$ is determined as 0.4145 with the lower and upper bounds of 0.3692 and 0.4597 , 
respectively. The error difference is $\pm 10.93 \%$. The damping coefficient $\left(\mathrm{c}_{\mathrm{PA}}\right)$ of the passenger is calculated using Eq. (6) as $2524.3 \mathrm{~kg} / \mathrm{s}$.

The error difference of the coefficients using the two sets of data is shown in the last column in Table 1, it is evident that the differences are small. Comparing the statistics results of SSE, R-square and RMSE in Table 1, an improved fitted curve is achieved using the reduced frequency range data than the one using the full frequency range. Thus, the parameter identification procedure using the curve fitting based on experimental data within the frequency range reduced to the vicinity of the resonance peak, instead of carrying out the test for the whole frequency range, can be proposed. The tests conducted show that such a reduction of the number of experimental data without increasing the standard deviation considerably lead to better curve fitting results.

Table 1. The curve fitting results of coefficients with the statistics for the car- passenger system

\begin{tabular}{|l|l|l|l|}
\hline Coefficients & Full frequency range & Reduced frequency range & Difference (\%) \\
\hline $\mathrm{k}_{\mathrm{PA}}[\mathrm{kN} / \mathrm{m}]$ & 115.6 & 115.9 & 0.2 \\
& $(105.6,125.5)$ & $\begin{array}{l}(106.3,125.5) \\
( \pm 8.28 \%)\end{array}$ & \\
& $( \pm 11.24 \%)$ & 0.4145 & 1.57 \\
\hline$\xi_{\mathrm{PA}}$ & 0.421 & $(0.3692,0.4597)$ & \\
& $(0.3736,0.4684)$ & $( \pm 10.93 \%)$ & 1.44 \\
\hline $\mathrm{c}_{\mathrm{PA}}[\mathrm{kg} / \mathrm{s}]$ & $( \pm 11.26 \%)$ & 2524.3 & \\
\hline $\mathrm{SSE}$ & 0.4334 & 0.1112 & \\
\hline R-square & 0.9807 & 0.981 & \\
\hline RMSE & 0.1291 & 0.1112 & \\
\hline
\end{tabular}

\section{Conclusions}

A damped single degree of freedom model and a damped two degree of freedom model of an elevator car system were used to identify the stiffness and damping characteristics of an elevator car system. With one passenger load on the platform, the passenger stiffness and damping coefficients are determined based on the parameters investigated from the empty platform system. The proposed identification procedure is designed to develop a model to predict the effect of the passenger behaviour on the dynamic response of the elevator car system with good accuracy and repeatability. The model will facilitate a study into the optimal choice of the damping and stiffness characteristics of the elevator car system. The predicted results will be used as benchmarks for further work to develop a software simulation tool for assessing the mechanical behaviour of elevator car systems taking into account the dynamic effects introduced by passenger loads.

\section{References}

[1] ISO 2003, Measurement of lift ride quality. ISO 18738: 2003(E), (2003)

[2] I. Herrera and S. Kaczmarczyk: $7^{\text {th }}$ International conference on Modern Practice in stress and Vibration Analysis, Journal of Physics: Conference Series 181 (2009)

[3] I. Herrera: Elevator Technology, 17, 152-9, (2008)

[4] I. Herrera: Elevator Technology, 17, 160-8, (2008)

[5] C. Silva: Vibration, Damping, Control and Design, London CRC Press, (2007)

[6] S. Rao: Mechanical Vibrations, Pearson Prentice Hall, (2004)

[7] MATLAB: Curve Fitting Toolbox 2.1, The MathWorks. 